\title{
ARCS IN X-RAY SELECTED CLUSTERS
}

\author{
I.M. GIOIA ${ }^{1,2}$, G.A. LUPPINO ${ }^{1}$, J. ANNIS ${ }^{1,3}$, F. HAMMER HA, $^{4,5}$ \\ AND O. LE FEVRE ${ }^{4,5}$ \\ ${ }^{1}$ Insitute for Astronomy, University of Hawaii, USA; \\ ${ }^{2}$ Istituto di Radioastronomia del CNR, Bologna, ITALY; \\ ${ }^{3}$ Fermilab, Batavia, IL, USA; \\ ${ }^{4}$ DAEC, Observatoire de Paris, Meudon, FRANCE \\ AND \\ ${ }^{5}$ CFHT Corporation, Kamuela, Hawaii, USA
}

\begin{abstract}
We present the results of an imaging survey to search for gravitationally lensed arcs in a sample of 40 EMSS clusters of galaxies. The lensing frequency obtained suggests that the X-ray selection is the preferred method for finding true, massive clusters which are more likely to exhibit the lensing phenomenon.

\section{Introduction}

Giant arcs are images of distant galaxies, gravitationally distorted by massive foreground clusters. Arc redshifts are systematically measured to be greater than the cluster redshifts (see among others Soucail et al. 1988; Pelló et al. 1992) and very similar to those of galaxies found in deep redshift surveys. This fact, combined with the symmetry and locations of the arcs, confirm the gravitational lensing idea. As the physics of the lensing is understood, we may use the properties of the arcs to probe the mass distribution in rich clusters. Another promising field of investigation is the study of the properties of very distant normal galaxies which are the "source" of the lensing. A large number of clusters needs to be observed to derive global properties of either the lens or the sources and large amounts of telescope time need to be allocated for both imaging and spectroscopy. A high efficiency procedure to search for arcs is then required.
\end{abstract}


We have been involved in an observational program to image a sample of 40 of the highest X-ray luminosity Extended Medium Sensitivity Survey (EMSS; Gioia et al. 1990) clusters with $\mathrm{L}_{x}>2 \times 10^{44} \mathrm{ergs} / \mathrm{s}$ and with redshifts in the range $0.15 \leq z \leq 0.83$, in order to search for gravitational lensing (Gioia and Luppino, 1994; Luppino et al. 1994). We assume that the hot, X-ray emitting gas is a tracer of the total gravitational potential of the cluster, both visible and dark matter. High X-ray luminosity is therefore a sign of a deep potential well and thus is an indicator of a true massive cluster, likely to exhibit the lensing phenomenon.

\section{Observations}

CCD images of the clusters were obtained during the period from May of 1992 through January of 1994 using the University of Hawaii $2.2 \mathrm{~m}$ telescope equipped with a Tektronix $2048 \times 2048$ CCD. $R$ band images of all 40 clusters, and $B$ band images of some of the clusters were acquired during 8 observing runs in photometric conditions and in excellent seeing (median seeing value of $0^{\prime \prime} .8 \mathrm{FWHM}$ in $R$ and $0^{\prime \prime} .9 \mathrm{FWHM}$ in $B$ ). Additional $V$ and $I$ images of a few clusters using both the UH $2.2 \mathrm{~m}$ and the CFHT were taken. The image scale was $0^{\prime \prime} .22 /$ pixel and the field of view was $7^{\prime} .5 \times 7^{\prime} .5$ $\left(1.54 h_{50}^{-1} \mathrm{Mpc} \times 1.54 h_{50}^{-1} \mathrm{Mpc}\right.$ at $z=0.15$ and $3.74 h_{50}^{-1} \mathrm{Mpc} \times 3.74 h_{50}^{-1} \mathrm{Mpc}$ at $z=0.82$ ). Although gravitationally lensed arcs are known to be relatively blue, they are still more easily detected in the $R$ band since their average $B-R$ color is roughly 1.3 (Soucail 1992). The additional $B$ or $V$ band images were taken to allow us to recognize any gravitational arcs by their relative blue color compared to the red cluster galaxies.

\section{Results and Discussion}

There is evidence of strong gravitational lensing in the form of giant arcs (length $l \geq 8^{\prime \prime}$, axis ratio $A \geq 10$ ) in 8 of the 40 clusters. Two additional clusters contain shorter arclets, and 6 more clusters contain candidate arcs that require follow-up observations to confirm their lensing origin. Luppino et al. (1994) present a fully description of the Hawaii arc survey and discuss the results obtained. We give here a brief summary of the resulting lensing frequency which is $20 \%$ for giant arcs, $25 \%$ for "secure" cases of lensing, and may be as high as $40 \%$ if all the candidate arcs prove to be real. There is a trend for giant arcs to be contained in the higher X-ray luminosity clusters $\left(L_{x}>10^{45} \mathrm{erg} \mathrm{s}^{-1}\right)$, thereby confirming our hypothesis that high X-ray luminosity does identify the most massive systems more likely to exhibit lensing. Figure 1 is a mosaic of eight EMSS clusters, seven of which contain giant arcs. The top two are MS0302.7+1658 (arc first reported in Girauud, 1991; see Mathez et al. 1992 for a complete study) and 
MS0451.6-0305 (discovered by Luppino et al. 1994). The following clusters are (from left in clockwise order) MS0440+0204 (Luppino et al. 1993); MS1006.0+1202 Le Fèvre et al. 1994); MS1455.0+2232 (arclet candidate, Smail et al. 1994a,b); MS2137.3-2353 (Fort et al. 1992); MS2053.7-0449 (Luppino and Gioia, 1992); MS1621.5+2640 (Luppino and Gioia, 1992).

The results from our survey are consistent with the predictions by several authors that compact lenses produce more arcs (Hammer, 1991; Wu and Hammer, 1993). The high rate of occurrence of arcs (eight out of forty clusters contain giant arcs corresponding to a frequency of $20 \%$ ), combined with the observed geometry of the arcs, indicate that the cluster central mass density profiles must be compact (Hammer, 1991, Hammer et al. 1993, Grossman and Saha 1994). There is also evidence of mass substructure in the central cluster cores as indicated by the presence of several arcs with large radii of curvature and from the observed orientation of the lensed arcs. We refer the reader to Luppino et al. 1994 for a comprehensive discussion of the results presented here.

We are presently obtaining spectra of the newly discovered arcs with the $\mathrm{CFH}$ and Keck telescopes to confirm the lensing hypothesis. Deep imaging observations with HST and X-ray observations with ROSAT and ASCA are also planned. The multiwavelength approach will allow to probe the structures of the sources and the state of the cluster X-ray gas.

This work received partial financial support from NSF Grant AST91199216 and NASA Grants NAG5-1752, NAG5-1880, and NAG5-2587. The UH CCD cameras were constructed using NSF Grant AST-9020680.

\section{References}

Fort, B., Le Fèvre, O., Hammer, F., Cailloux, M. 1992, ApJL, 399, L125.

Gioia, I. M., Maccacaro, T., Schild, R.E., Wolter, A., Stocke, J.T., Morris, S. L., Henry, J. P. 1990a, ApJS, 72, 567.

Gioia, I.M., and Luppino, G. A. 1994, $A p J S$, in press.

Giraud, E., 1991, ESO Messenger, 66, 65.

Grossman, S. \& Saha, P. 1994, ApJ, in press.

Hammer, F. 1991, ApJ, 383, 66.

Hammer, F., Angonin-Willaime, M.C., Le Fèvre , O., Wu, X. P., Luppino, G. A., and Gioia, I. 1993, in Gravitational Lenses in the Universe, 31st Liege Int. Astroph. Colloquium 1993.

Luppino, G.A., and Gioia, I.M. 1992, $A \cup A, 265$, L9.

Le Fèvre , O., Hammer, F., Angonin, M.C., Gioia, I., and Luppino, G. A., 1994, ApJL, 422, L5.

Luppino, G.A., Gioia, I.M., Annis, J., Le Fèvre , O., and Hammer, F. 1993, ApJ, 416, 444.

Luppino, G.A., Gioia, I.M., Annis, J., Le Fèvre , O., and Hammer, F. 1994, ApJ, submitted.

Mathez, G., Fort, B., Mellier, Y., Picat, J.-P., Soucail, G. 1992, A\&A, $256,343$.

Pelló, R., Le Borgne, J.F., Sanahuja, B., Mathez, G., Fort, B., 1992, A\&A, 266, 6.

Smail, I., Ellis, R., and Fitchett, M. 1994a, MNRAS, in press. 
Smail, I., Ellis, R., Fitchett, M., and Edge, A. 1994b, MNRAS, in press.

Soucail, G., Mellier, Y., Fort, B., Mathez, G., and Cailloux, M., 1988, $A \& A, 191, \mathrm{~L} 19$.

Soucail, G. 1992, in Clusters and Superclusters of Galaxies, ed. Fabian, NATO ASI Series Vol. 336, 199.

Wu, X. P., and Hammer, F. 1993, MNRAS, 262, 187.

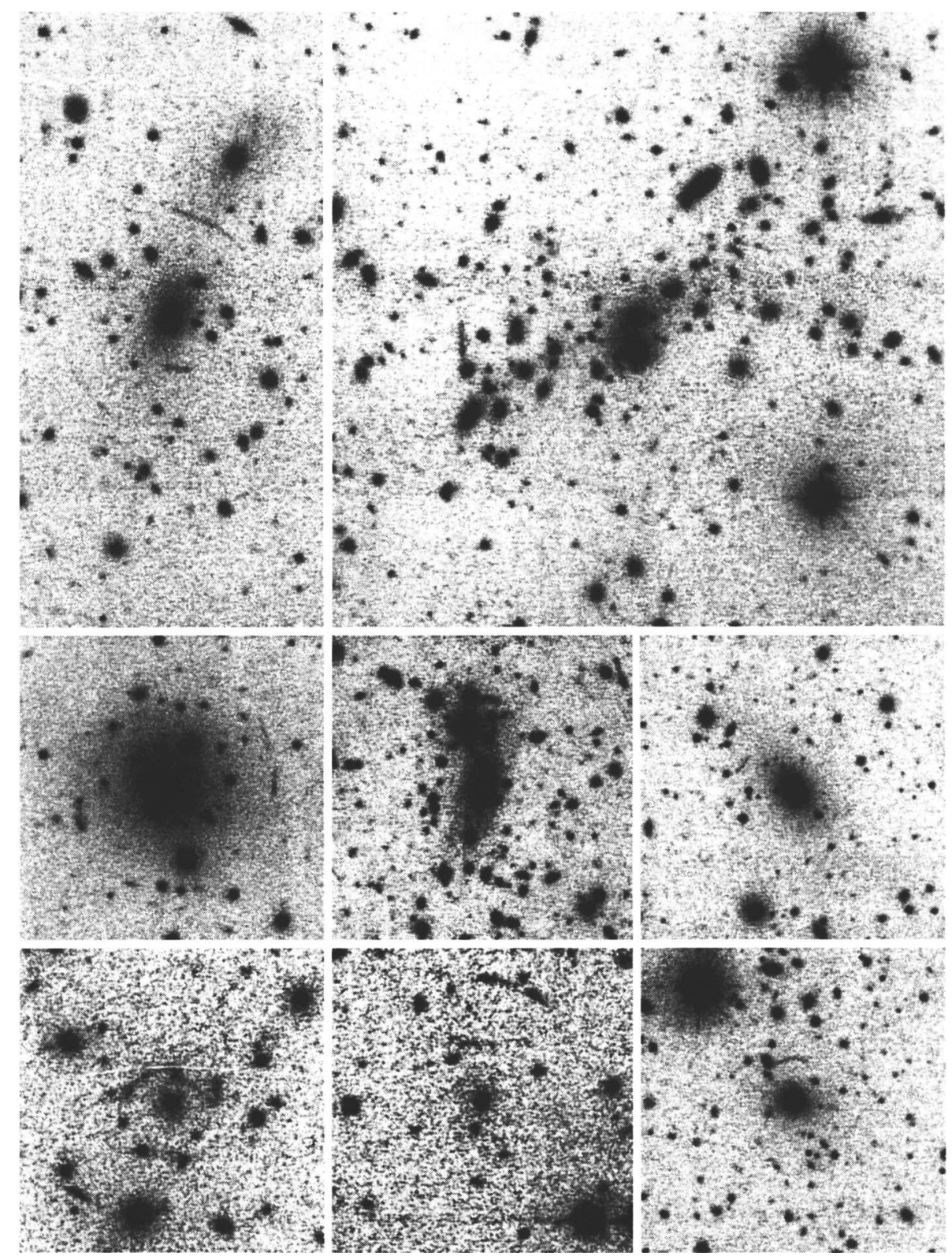

Figure 1 - CCD mosaic of 8 EMSS clusters containing arcs. 